\title{
Buchbesprechung:
}

\section{Essentials of Soil Science - Soil formation, functions, use and classification (World Reference Base, WRB)}

Winfried E.H. Blum, Peter Schad, Stephen Nortcliff, 2017. 171 Seiten, 101 Abbildungen, 22 Tabellen, $17 \times 24 \mathrm{~cm}$, softcover, ISBN 978-3-443-01090-4, 27,90 EUR. Weitere Informationen unter: Borntraeger-cramer.com/9783443010904

\author{
Buchbesprechung verfasst von: \\ Martin H. Gerzabek
}

Universität für Bodenkultur Wien (BOKU), Institut für Bodenforschung, Peter-Jordan-Straße 82, 1190 Vienna, Österreich, martin.gerzabek@boku.ac.at

Schon das Cover des Buches verrät, dass dieses Buch eine enge Verwandtschaft mit dem Klassiker „Bodenkunde in Stichworten" aufweist, der von Winfried Blum ebenfalls bei Borntraeger herausgegeben wird. Das Buch „Bodenkunde in Stichworten“ aus der Reihe „Hirts Stichwörterbücher" ist nun seit fast fünf Jahrzehnten in der bereits 7. Auflage ein Klassiker auf dem Gebiet der Lehrbücher der Bodenkunde und diente Generationen von Studierenden und SchülerInnen als leicht lesbare und übersichtliche Einführung. Die Entscheidung, eine deutlich bearbeitete und erweiterte Version in Englisch herauszugeben, liegt auf der Hand. Mit Peter Schad und Stephen Nortcliff konnten zwei international sehr angesehene Wissenschafter als Koautoren gewonnen werden.

Die Bodenkunde wird zunehmend in vielen verwandten Wissenschaftsgebieten zur unverzichtbaren Stütze. Immer mehr Wissenschafter, Studierende und Fachinteressierte haben die Notwendigkeit sich mit dem Thema vertraut zu machen. Genau hier setzt das Buch an. Es gibt einen fundierten Überblick und eine leicht lesbare Einführung in die Bodenkunde. Nach einer kurzen Einleitung folgen die Bodenbestandteile, die physikalischen, chemischen und biologischen Bodeneigenschaften, die Prozesse der Bodenbildung, die Bodenklassifikation, sowie die Rolle des Bodens in der Umwelt. In letzterem Kapitel gehen die Autoren auf die Bodenfunktionen, deren Konkurrenz und Gefährdung sowie den Bodenschutz ein. Der Boden als Pflanzenstandort wird als eigenes Kapitel präsentiert, wie auch Bodeninformationssysteme und Geschichte der Bodenkunde. Im Kapitel Bodensystematik konzentrieren sich die Autoren sachgerecht für ein englischsprachiges Buch auf die international anwendbare WRB (World Reference Base of Soil Resources) ohne auf andere Systematiken zu vergessen. Das Kapitel sticht durch die exzellenten Fotos von Bodenprofilen aus aller Welt samt Beschreibungen hervor.

Aus meiner Sicht kann das Buch uneingeschränkt empfohlen werden für all jene, die eine leicht lesbare aber fundierte Einführung in die Bodenkunde auf Englisch suchen. Gerade auch in der universitären Lehre sehe ich da große Potenziale für jene, die sich auf einen Auslandsaufenthalt bzw. englischsprachige Lehrveranstaltungen vorbereiten, bzw. jene internationale Studierende, die sich rasch in die Materie einlesen wollen. 